\title{
Element Excitation Optimization for Phased Array Fault Diagnosis
}

\author{
Prajosh K P, Uday K Khankhoje, and Francesco Ferranti
}

\begin{abstract}
We present techniques for solving the problem of detecting element failures in phased array antennas by using a combination of a single fixed probe and an optimization of element excitations using principles derived from compressive sensing. This departs significantly from conventional techniques where the excitations are held constant and probes are instead moved spatially to collect measurements. Doing so helps us to accomplish two objectives with regards to successful fault diagnosis. First, we achieve a reduction in the number of measurements required compared to the state of the art; this reduction is particularly significant in the case of highnoise measurements where existing methods fail. Second, our techniques solve the problem of fault diagnosis in the case of real valued measurements (i.e. intensity measurement along with phase detection instead of phase measurement), which leads to simpler measurement hardware. We use nonconvex optimization algorithms to generate numerical results in support of our conclusions.
\end{abstract}

Index Terms- Compressive sensing, antenna arrays, fault diagnosis

\section{INTRODUCTION}

Phased array antennas are widely used in wireless communications, remote sensing, and radar applications [1]. Future deployments of telecommunications [2] and automotive radars [3] will extensively rely on the proper functioning of such systems. The failure of one or more antenna elements can severely impair the system operation, making the monitoring of the health of these systems increasingly critical. In particular, methods of fault diagnosis that can work in situ with minimal modification are particularly appealing, rather than those that require systems to be taken inside specialized test facilities.

In this Paper, we propose techniques of fault diagnosis that rely on a single fixed probe, and can work with complex or real valued measurement data in the near and far-field. Since we do not require probes to scan the array under test (AUT) from different spatial locations, the techniques are in-principle compatible with in situ measurements of the AUT in operational settings, provided that scattering from the neighbouring environment can be minimized

The typical use-case scenario for fault diagnosis considered in recent literature has centred around element failure of a small fraction of the total element number. In this scenario, the principles of compressive sensing (CS) [4] have offered

Prajosh K P and U K Khankhoje (corresponding author, email: uday@ee.iitm.ac.in) are with the Department of Electrical Engineering, Indian Institute of Technology Madras, Chennai, India, and F Ferranti is with the Institut Mines-Télécom Atlantique, CNRS UMR 6285 Lab-STICC, Brest, France. the promise of fault diagnosis by making much fewer measurements than the number of antennas in the array [5]. Several variations on this theme have been studied, such as fault diagnosis from near [5] or far [6] field data, and from measurements with [7] and without [8] phase. It is known that for CS techniques to work, the sensing matrix must satisfy the so-called "Restrictive isometry property" (RIP) [9]. By choosing the measurement locations in a particular way [6], it was shown that the sensing matrix was a random partial Fourier matrix, which in turn satisfied RIP provided sufficient measurements were made.

Typically, the RIP property is difficult to verify in practise, and often the CS machinery is used in a heuristic manner by resorting to random measurement locations [8]. Instead of the RIP, it is known that minimizing the mutual coherence of the sensing matrix provides (weaker) guarantees of convergence [10]; recent work has shown how this idea can be applied to electromagnetic compressive sensing applications [11]. Returning to the theme of fault diagnosis in phased arrays, we note that in all the work referred to so far, the sensing matrix was generated by choosing different sensing locations in space. This imposes severe restrictions on how suitable the sensing matrices are for applying CS principles.

Recent work [12], [13] has shown an elegant method of fault diagnosis where the probe locations are kept fixed and the phase of the element excitations are varied to generate a series of measurements. The phases were chosen at random and fault diagnosis was accomplished by solving an unconstrained $\ell_{p}$ minimization problem $(0<p \leq 1)$. We extend this idea further by allowing the entire sensing matrix to be subject to design. In particular, we allow both the amplitude and phases of the element excitations to be optimized in order to minimize the mutual coherence of the sensing matrix. The theory of compressive sensing tells us that by doing so, the number of measurements required for successful fault diagnosis can be reduced, much more so in the average case as compared to the worse case [14, Ch. 7].

In our work we consider two types of measurements, depending on the type of hardware available. If we represent the phasor of a time-harmonic electromagnetic field by $r e^{j \theta}$, where $r$ and $\theta$ represent the amplitude and phase, respectively, then we refer to a complex measurement as one which measures both the amplitude $(r)$ and the phase $(\theta)$ via measurements of the in-phase and quadrature components. We refer to a real measurement as one which measures the amplitude $(r)$ and the sign of the in-phase component $(\operatorname{sgn}(\cos \theta))$; this can be implemented by using a power meter (measurement $\propto r^{2}$ ) and a phase detector to determine the sign. Since the latter does not actually measure the phase, a real measurement requires 
simpler hardware than a complex measurement and offers the possibility of cheaper hardware for large scale Internet of Things (IoT) type of applications. In our approach, the same technique can be used for solving both problems without any approximations, and the only difference lies in the nature of the sensing matrix that is employed. This is accomplished in the real measurements case by a specific choice of the element excitation phase which will be detailed subsequently. This confers a great deal of generality to our approach, not previously offered by other approaches. We note that there exists a related problem of fault diagnosis using intensity only measurements (e.g. [8]); however, we do not address this problem in the current work.

This Paper is organized as follows: we formulate the problem mathematically in Section III. where we also describe the solution strategies. In Section III, we present numerical results in support of the efficacy of our technique for complex and real measurements along with their analysis. Finally, we conclude in Section [V] with a summary of our results.

\section{Problem Formulation and Methods}

\section{A. Problem setup}

We consider the measured far-field, $E(\theta)$, from a one dimensional $N$-element array of elements spaced uniformly by a distance $d$ as:

$$
E(\theta)=\sum_{n=1}^{N} E_{n}(\theta) x_{n} \rho_{n} \exp (j k n d \cos \theta),
$$

where $k$ is the wavevector, $\theta$ is the angle measured from the array axis, and $E_{n}, x_{n}, \rho_{n}$ are the element pattern, excitation, and fault state ( 1 if the element is working, and 0 if it has failed) of the $n^{\text {th }}$ element, respectively. The expression can easily be generalized for planar arrays or near field measurements.

We first sketch the conventional scheme of fault diagnosis where measurements are taken at different locations in space. Assuming for simplicity that the elements are identical (with pattern $E_{0}$ ), and $M$ measurements are taken at locations: $\left\{\theta_{m}\right\}_{m=1}^{M}$, Eq. 11 becomes:

$$
\tilde{y}=A \tilde{x}, \quad A_{m n}=E_{0} \exp \left(j n k d \cos \theta_{m}\right),
$$

where $\tilde{y} \in \mathbb{C}^{M}, \tilde{x} \in \mathbb{C}^{N}$ (with $\tilde{x}_{n}=x_{n} \rho_{n}$ ) represent the vectors of measurements and sensor excitations, respectively, and $A \in \mathbb{C}^{M \times N}$ is the system sensing matrix. Evidently, the only control over designing the system matrix comes by adjusting the measurement locations, $\theta_{m}$.

However, Eq. (1) can also be recast in the following way, with an allowance for using different excitations for each measurement at the fixed observation angle, $\theta$, as:

$$
\tilde{y}=B \rho, \quad B_{m n}=E_{0} \exp (j n k d \cos \theta) x_{m n},
$$

where $x_{m n}$ is the excitation of the $n^{\text {th }}$ element for the $m^{\text {th }}$ observation. Since the element excitations have entered the system matrix, one can aim to improve the efficiency of fault diagnosis by optimizing the element excitations; this will be demonstrated shortly.

To complete the connection with compressive sensing, we take recourse to the measurements obtained from the same array but without faults, recorded in $\hat{y} \in \mathbb{C}^{M}$. Denoting the difference in measurements between the ideal and faulty array by $y=\hat{y}-\tilde{y}$, and under the assumption that the number of faults is a small fraction of the total element number, $N$, the problem that needs to be solved for fault diagnosis becomes:

$$
\min _{z}\|z\|_{0} \text {, s.t. }\|y-B z\|_{2}<\eta
$$

where $z=\mathbf{1}-\rho$ (with $\mathbf{1} \in \mathbb{R}^{N}$ being a vector of ones) and $\eta$ is a small scalar proportional to the variance of measurement noise. We have defined the problem statement for far field measurements with identical elements for ease of illustration; we emphasise that our methods are completely general, and show in the Appendix how to deal with arrays that might be heterogeneous and measured in the near field.

\section{B. Solution philosophy and methodology}

As the name 'phased array' suggests, the element excitations have phase and or amplitude control, thus providing a larger canvas to design the sensing matrix than is possible by adjusting receiver positions only.

It is known that the mutual coherence, $\mu$, of a general $M \times N$ fat matrix is lower bounded by the Welch bound, $\sqrt{\frac{N-M}{M(N-1)}} \leq \mu \leq 1$, and that alternating projection algorithms can be used for designing matrices which approach this bound [15][16]. Of great interest is the improvement in performance (e.g. a reduction in the number of measurements) on account of a reduction in $\mu$. Worst case analysis, which tends to be rather pessimistic, says that a signal can be reconstructed from compressive measurements only if it has a sparsity factor (i.e. its 0 -norm) up to $O\left(1+\frac{1}{\mu}\right)$; on the other hand, when average case performance is sought, the sparsity factor can be up to $O\left(\frac{1}{\mu^{2}}\right)$ [14, Thm. 7.3]. This means that for a given number of measurements, an array with a greater number of faults can be detected (on average) if the sensing matrix is optimized (for lower mutual coherence). The idea of optimizing the mutual coherence of the sensing matrix and not using a random matrix is the key idea of our work, and one of the distinguishing features compared to recent literature [13]. This allows a reduction of the needed number of measurements for a successful fault diagnosis. This reduced number can be an important factor in multiple scenarios, for e.g. : (1) when the forward model used to compute the radiation pattern has a non-negligible computational cost when changing excitation signals (e.g., see reconfigurables reflectarray antennas in [17]), and (2) when the fault diagnosis performed in an onsite way has to minimally affect the operation time of the antenna array. The numerical results in the subsequent Section amply demonstrate the power of our approach.

Often, the nonconvex, combinatorial $\|z\|_{0}$ function in Eq. (4) above is relaxed to $\|z\|_{1}$, since this is the closest convex function to the $\ell_{0}$ function. Another approach that is considered is 
the relaxation to the nonconvex function given by $\|z\|_{p}$ with $0<p<1$. Although this nonconvex relaxation does not come with convergence guarantees, it has been empirically found to outperform the $\ell_{1}$ convex relaxation [13], [18]. These approaches are summarized in terms of the following unconstrained optimization problem to be solved for fault diagnosis:

$$
\min _{z}\|y-B z\|_{2}+\lambda\|z\|_{p}, 0<p \leq 1,
$$

where $\lambda$ is an empirical hyperparameter.

In our work, we assume that the phase and amplitude of the phased array is quantized to 6 bits each to reflect realistic hardware for phase shifters. In implementing related work [13], we generate the amplitude ( 0 to 1$)$ and phase ( 0 to $2 \pi$ ) by drawing from the multinomial probability distribution function. In our optimized matrix approach, we initialize with the earlier random matrix, then apply an alternating projection procedure [15][14, Ch. 2], and end by quantizing the amplitude and phase. The behaviour of the mutual coherence values of the matrices used in the results below are shown in Fig. 1. It can be seen that even after quantization, the optimized matrices closely approach the Welch bound (attained by Grassmanian matrices).

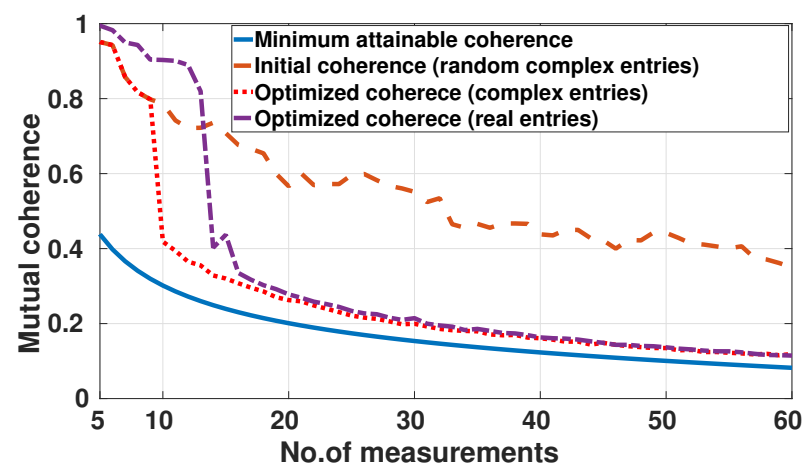

Fig. 1. Mutual coherence values for $M \times 100$ matrices for random, optimized, and real sensing matrices. The amplitude and phase of every matrix element has been quantized to 6 bits each (for the complex entries)

\section{Approach for real measurements}

For the case of optimizing the sensing matrix for real measurements, we restrict the phase to only 1 bit (i.e. a phase of 0 or $\pi$ ), but allow 6 bits of amplitude control. As an illustration, it can be seen from Eq. (3) that if the element patterns are real valued and measured at $\theta=\frac{\pi}{2}$, then by keeping the excitations real valued, the measured field is also real valued, and thus no information is lost by a using a sensor capable of only real-valued measurements. This motivates fault diagnosis using the existing scheme by restricting the phase to 1-bit for the real-measurements case. We show in the Appendix how to overcome the restriction of real valued element patterns and $\theta=\frac{\pi}{2}$ for a completely general antenna array. In the next Section, we provide details of the algorithms used and the results obtained.

\section{RESUlTS \& ANALYSIS}

Algorithms: In this paper, we present the solution to the fault diagnosis problem by three strategies:

(i) a solution to the original problem in Eq. (4) by means of a greedy pursuit algorithm, the Compressive Sampling Matching Pursuit (CoSaMP) [19],

(ii) and (iii) A solution to the relaxed convex and nonconvex problem in Eq. (5) with $p=1$, and $0<p<1$, respectively, by means of an iteratively reweighted $\ell_{1}$ (IRL1) minimization algorithm [20] implemented via the alternating direction method of multipliers (ADMM) [21].

Error metrics: We report fault diagnosis success according to a metrics computed on the recovered solution vector, $\rho$, the rate of successful recovery (RSR). While [13] defines RSR in terms of the mean squared error $(\mathrm{MSE})<-30 \mathrm{~dB}$, we adopt a more explicit criteria, and define RSR- $k$ as the number of measurements such that $k \%$ of trials give a perfect reconstruction of the fault state of the array; we implement a thresholding function on the reconstructed vector prior to computing the RSR (i.e. if $\rho_{n}<0.5$, set $\rho_{n}=0$, else set $\left.\rho_{n}=1\right)$. In our simulations, we consider 200 Monte Carlo trials for every result shown.

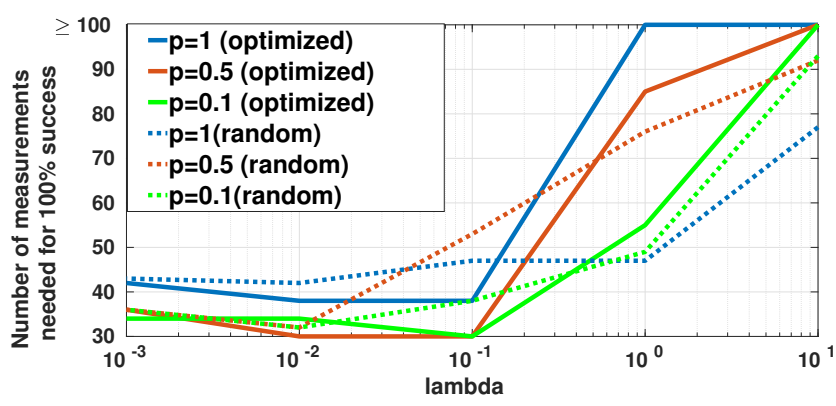

Fig. 2. Performance of IRL1 on a 100 element linear array for different values of the hyperparameter, $\lambda$ for the case of (complex) random and optimized sensing matrices. The number of faults and SNR are fixed at 10 and $20 \mathrm{~dB}$ respectively.

Implementation details: Note that CoSaMP requires a priori information of the expected sparsity of the solution vector. Since this information is typically not known, we run the algorithm with various guesses for the solution sparsity. Of those, we pick the sparsity corresponding to the smallest residual error.

In the $\ell_{p}$ norm relaxation algorithm, we determine the $\lambda$ hyperparameter of Eq. (5) empirically using a grid search. A study of this search is shown in Fig. 2 where the value of $\lambda$ is varied logarithmically. We find that $\lambda=0.1$ worked the best for optimized matrices, while for random matrices $\lambda=0.01$ was optimal. The termination criteria used in CoSaMP and IRL1 is a limit on the total number of iterations (500 in our case). For every result, we perform 200 Monte Carlo iterations; simulations were performed on an Intel Core i7$8700 \mathrm{CPU} 3.20 \mathrm{GHz} \times 12$ processor and took $45-50$ seconds on average. 
Comparative results for varying fault numbers: We consider a 100-element linear array consisting of ideal isotropic radiators with measurements at $\theta=\frac{\pi}{2}$ in the far field (noise corrupted with a signal to noise ratio (SNR) of $10 \mathrm{~dB}$ ). The centerpiece of our results is Table I] where varying numbers of faults are diagnosed by a variety of methods, including our optimized matrix approach, the random matrix approach from [13], as well as the real-measurements case.

\begin{tabular}{|c|c|c|c|c|c|c|c|c|c|c|c|c|c|c|}
\hline \multirow{2}{*}{$\mathrm{s}$} & \multicolumn{1}{|c|}{ IRL1 $p=1$} & \multicolumn{2}{c|}{ IRL1 $p=0.5$} & \multicolumn{2}{c|}{ IRL1 $p=0.1$} & \multicolumn{5}{|c|}{ CoSaMP } \\
\cline { 2 - 14 } & $\mathrm{R}$ & $\mathrm{O}$ & $\mathrm{E}$ & $\mathrm{R}$ & $\mathrm{O}$ & $\mathrm{E}$ & $\mathrm{R}$ & $\mathrm{O}$ & $\mathrm{E}$ & $\mathrm{R}$ & $\mathrm{O}$ & $\mathrm{E}$ \\
\hline \multicolumn{10}{|c|}{ Number of measurements for RSR-100 $\downarrow$} \\
\hline 1 & 13 & 11 & 15 & 10 & 10 & 15 & $\mathbf{9}$ & $\mathbf{9}$ & 14 & 34 & 15 & 27 \\
\hline 5 & 58 & 39 & 49 & 61 & 36 & 56 & 59 & $\mathbf{2 5}$ & 64 & 79 & 48 & 63 \\
\hline 10 & $\diamond$ & 69 & 79 & $\diamond$ & $\mathbf{6 1}$ & 80 & $\diamond$ & 63 & 91 & 99 & 70 & 84 \\
\hline 15 & $\diamond$ & 80 & 99 & $\diamond$ & $\mathbf{7 1}$ & 94 & $\diamond$ & 79 & 98 & $\diamond$ & 80 & 99 \\
\hline \multicolumn{10}{|c|}{ Nomber of measurem nts for RSR-90 $\downarrow$} \\
\hline 1 & 9 & 9 & 10 & $\mathbf{6}$ & $\mathbf{6}$ & 10 & $\mathbf{6}$ & $\mathbf{6}$ & 10 & 13 & 10 & 14 \\
\hline 5 & 34 & 30 & 36 & 28 & 20 & 34 & 30 & $\mathbf{1 9}$ & 40 & 47 & 34 & 50 \\
\hline 10 & $\diamond$ & 53 & 59 & $\diamond$ & 43 & 63 & $\diamond$ & $\mathbf{3 8}$ & 68 & 77 & 58 & 66 \\
\hline 15 & $\diamond$ & 67 & 77 & $\diamond$ & $\mathbf{6 1}$ & 79 & $\diamond$ & 65 & 86 & 97 & 67 & 82 \\
\hline
\end{tabular}

\section{TABLE I}

RESULTS SHOWING THE NUMBER OF MEASUREMENTS REQUIRED TO ACHIEVE RSR-100 (TOP ROWS) AND RSR-90 (BOTTOM ROWS) FOR THE DIFFERENT ALGORITHMS CONSIDERED IN THIS PAPER (IN COLUMNS) AS A FUNCTION OF NUMBER OF FAULTS, $s$, FOR AN ARRAY OF 100 ELEMENTS AND AN SNR OF 10 DB. ENTRIES IN BOLDFACE CORRESPOND TO THE BEST RESULTS FOR THAT PARTICULAR VALUE OF $s$ AND RSR- $k$. R,O,E REFER TO RANDOM, OPTIMIZED, AND REAL MATRICES, RESPECTIVELY.

THE SYMBOL $\diamond$ REFERS TO CASES WHERE THE NUMBER OF MEASUREMENTS EXCEED THE NUMBER OF ELEMENTS (N) AND COMPRESSIVE SENSING IS NO LONGER MEANINGFUL.

Comparative results for varying SNR: Next, in Table [I] we consider the same array as above, except that we fix the number of faults to 10 , and vary the SNR on the measurements.

\begin{tabular}{|c|c|c|c|c|c|c|c|c|c|c|c|c|}
\hline \multirow{2}{*}{ SNR } & \multicolumn{1}{|c|}{ IRL1 $p=1$} & \multicolumn{2}{|c|}{ IRL1 $p=0.5$} & \multicolumn{3}{|c|}{ IRL1 $p=0.1$} & \multicolumn{5}{|c|}{ CoSaMP } \\
\cline { 2 - 14 } & $\mathrm{R}$ & O & $\mathrm{E}$ & $\mathrm{R}$ & $\mathrm{O}$ & $\mathrm{E}$ & $\mathrm{R}$ & $\mathrm{O}$ & $\mathrm{E}$ & $\mathrm{R}$ & $\mathrm{O}$ & $\mathrm{E}$ \\
\hline \multicolumn{10}{|c|}{ Number of measurements for RSR-100 } \\
\hline 10 & $\diamond$ & 65 & 75 & $\diamond$ & $\mathbf{5 5}$ & 82 & $\diamond$ & 65 & 85 & 100 & 64 & 85 \\
\hline 20 & 40 & 38 & 47 & 31 & $\mathbf{2 9}$ & 43 & 33 & 30 & 45 & 34 & 30 & 42 \\
\hline 30 & 38 & 35 & 44 & 31 & $\mathbf{2 8}$ & 41 & 32 & 30 & 44 & 29 & 30 & 40 \\
\hline \multicolumn{10}{|c|}{ Number of measurements for RSR-90 $\downarrow$} \\
\hline 10 & $\diamond$ & 53 & 62 & $\diamond$ & 39 & 61 & $\diamond$ & $\mathbf{3 7}$ & 69 & 70 & 51 & 67 \\
\hline 20 & 36 & 32 & 40 & 28 & $\mathbf{2 4}$ & 36 & 28 & 26 & 39 & 27 & 26 & 35 \\
\hline 30 & 33 & 31 & 37 & 26 & $\mathbf{2 4}$ & 35 & 29 & 25 & 40 & 26 & 26 & 34 \\
\hline
\end{tabular}

TABLE II

RESULTS SHOWING THE NUMBER OF MEASUREMENTS REQUIRED TO ACHIEVE RSR-100 (TOP ROWS) AND RSR-90 (BOTTOM ROWS) FOR THE DIFFERENT ALGORITHMS CONSIDERED IN THIS PAPER (IN COLUMNS) AS A FUNCTION OF SNR, FOR AN ARRAY OF 100 ELEMENTS AND A FIXED NUMBER OF FAULTS, $s=10$. ENTRIES IN BOLDFACE CORRESPOND TO THE BEST RESULTS FOR THAT PARTICULAR VALUE OF SNR AND RSR- $k$. THE REST OF THE SYMBols ARE AS IN TABLE!

Comparative results for varying array size: Finally, in Table III we consider the performance of various algorithms against varying array sizes, keeping the number of faults and SNR fixed at 10 and $10 \mathrm{~dB}$, respectively.

Analysis: We now analyze the results that we have obtained, drawing out the salient features of our work.

1) The results in all the Tables strongly suggest that regardless of the algorithm used, the optimized matrix approach always

\begin{tabular}{|c|c|c|c|c|c|c|c|c|c|c|c|c|c|}
\hline \multirow{2}{*}{$N$} & \multicolumn{2}{|c|}{ IRL1 $p=1$} & \multicolumn{1}{|c|}{ IRL1 $p=0.5$} & \multicolumn{2}{|c|}{ IRL1 $p=0.1$} & \multicolumn{5}{c|}{ CoSaMP } \\
\cline { 2 - 13 } & $\mathrm{R}$ & $\mathrm{O}$ & $\mathrm{E}$ & $\mathrm{R}$ & $\mathrm{O}$ & $\mathrm{E}$ & $\mathrm{R}$ & $\mathrm{O}$ & $\mathrm{E}$ & $\mathrm{R}$ & $\mathrm{O}$ & $\mathrm{E}$ \\
\hline \multicolumn{10}{|c|}{ Number of measurements for RSR-100 $\downarrow$} \\
\hline 50 & $\diamond$ & 42 & $\diamond$ & $\diamond$ & 43 & $\diamond$ & $\diamond$ & $\mathbf{4 0}$ & $\diamond$ & $\diamond$ & 50 & $\diamond$ \\
\hline 70 & $\diamond$ & 51 & $\diamond$ & $\diamond$ & $\mathbf{4 6}$ & 68 & $\diamond$ & 57 & 69 & $\diamond$ & 52 & $\diamond$ \\
\hline 90 & $\diamond$ & 65 & 69 & $\diamond$ & $\mathbf{5 6}$ & 74 & $\diamond$ & 61 & 84 & 85 & 57 & 78 \\
\hline \multicolumn{10}{|c|}{ Number of measurements for RSR-90 $\downarrow$} \\
\hline 50 & $\diamond$ & 36 & 41 & $\diamond$ & $\mathbf{3 1}$ & 41 & $\diamond$ & 33 & 44 & $\diamond$ & 39 & 48 \\
\hline 70 & $\diamond$ & 43 & 52 & $\diamond$ & $\mathbf{3 3}$ & 53 & $\diamond$ & 35 & 51 & 63 & 44 & 58 \\
\hline 90 & $\diamond$ & 51 & 57 & $\diamond$ & 39 & 59 & $\diamond$ & $\mathbf{3 7}$ & 66 & 63 & 50 & 62 \\
\hline
\end{tabular}

\section{TABLE III}

RESULTS SHOWING THE NUMBER OF MEASUREMENTS REQUIRED TO ACHIEVE RSR-100 (TOP ROWS) AND RSR-90 (BOTTOM ROWS) FOR THE DIFFERENT ALGORITHMS CONSIDERED IN THIS PAPER (IN COLUMNS) AS A FUNCTION OF NUMBER ARRAY SIZE, $N$, FOR A FIXED NUMBER OF FAULTS, $s=10$, AND AN SNR OF 10 DB. ENTRIES IN BOLDFACE CORRESPOND TO THE BEST RESULTS FOR THAT PARTICULAR VALUE OF $N$ AND RSR- $k$. THE REST OF THE SYMBOLS ARE AS IN TABLE!

outperforms the random matrix approach under varying number of faults, noise levels, as well as different array sizes. The difference between the optimized and random matrix approaches becomes clearer as the number of faults increases. This is to be expected from the study of the mutual coherence (see Fig. 11, where an improvement as compared to the random matrix case is seen only after the number of measurements exceeds 9 (for an arrary of 100 elements).

2) We emphasize that our approach is particularly robust under high noise conditions; in particular, as the (10 dB SNR) results in Table I show, in most cases the random matrix approach fails (marked by a diamond symbol indicative of $>N$ measurements, at which point a brute force approach of testing each element individually is better). In general it is seen that the optimized matrix approach takes 1-2 times fewer measurements than the random sensing matrix case.

3) A remarkable observation from all the results is that the number of measurements required for the case of real measurements is comparable with that from the random sensing matrix case with complex measurements. In fact, in several instances the former succeeds, while the latter fails.

4) Among the methods considered in this paper, the IRL1 algorithm with $p=0.5$ delivers the best results, though the results with $p=0.1$ are only slightly worse in comparison. The results of the CoSaMP algorithm are also competitive.

\section{CONCLUSION}

In summary, we have presented a new framework in which fault diagnosis can be performed on phased arrays without taking recourse to moving detectors in space. Our approach optimizes the element excitations and leverages ideas from compressive sensing to perform this diagnosis, giving us two distinct advantages with respect to the most advanced stateof-the-art: the fault diagnosis can be done with (a) fewer measurements, and (b) in the presence of real measurements. We show the superior performance of our methods through extensive numerical simulations; in particular, we show how our methods are robust in the presence of noise, delivering results when other methods fail. Finally, our methods can easily 
be generalized to near field measurements of heterogeneous arrays.

\section{APPENDIX \\ HETEROGENEOUS ARRAYS, NEAR FIELD MEASUREMENTS}

We consider the case where the array elements are heterogeneous, and the (possibly near-field) measurements may be away from the normal direction (i.e. $\theta \neq \frac{\pi}{2}$ ). In this most general case (still ignoring mutual coupling, though), Eq. (1) gets generalized to:

$$
E(r, \theta)=\sum_{n=1}^{N} E_{n}\left(r_{n}, \theta_{n}\right) x_{n} \rho_{n} .
$$

For e.g., if a particular element were a Hertz dipole at the origin, and we were measuring the $\theta$-component of the field, $E_{\theta}(r, \theta)$, we would have $E_{n}\left(r_{n}, \theta_{n}\right)=$ $-j Z_{0} l e^{-j k r_{n}} /\left(4 \pi k r_{n}^{3}\right) \sin \theta_{n}$, where $l$ is the dipole length and $Z_{0}$ is free space impedance.

We define a matrix of excitations, $X \in \mathbb{C}^{M \times N}$ s.t. $X_{m n}=$ $x_{m n}$, a vector $t \in \mathbb{C}^{N}$ s.t. $t_{n}=E_{n}\left(r_{n}, \theta_{n}\right) \rho_{n}$, a vector $\tilde{t} \in \mathbb{C}^{N}$ s.t. $\tilde{t}_{n}=E_{n}\left(r_{n}, \theta_{n}\right)$, and a diagonal matrix $W \in \mathbb{C}^{N \times N}$ s.t. $W_{n n}=1 / E_{n}\left(r_{n}, \theta_{n}\right)$. Then the fault diagnosis optimization problem is similar to the earlier Eq. (4) with a redefinition of $z$ to guarantee its sparsity, as below:

$$
\min _{z}\|z\|_{0} \text {, s.t. }\|y-X z\|_{2}<\eta \text {, where } z=W(\tilde{t}-t) .
$$

With the identical reformulation of $z$, the nonconvex relaxation described by Eq. (5) can be used in this general setting. Thus, as desired, the system matrix $X$ can be completely optimized for fault diagnosis even in this general setting.

Real measurement scenario: In this case, the principle is to use the phase of the excitation, $x_{n}$, to cancel the phase of the accompanying $E_{n}\left(r_{n}, \theta_{n}\right)$ term of Eq. (6). While the phase is restricted in this manner (up to a sign), we are free to adjust the amplitude of the excitations to optimize the sensing matrix. As a result, the measurement, $E(r, \theta)$, is real valued. This approach depends on there being sufficient bits available for phase control to approximate the negative of the phase of $E_{n}\left(r_{n}, \theta_{n}\right)$.

\section{ACKNOWLEDGEMENTS}

The authors acknowledge useful discussions on array fault diagnosis with Rajat Dwaraknath, IIT Madras.

\section{REFERENCES}

[1] R. C. Hansen, Phased array antennas, vol. 213. John Wiley \& Sons, 2009.

[2] W. Hong, K.-H. Baek, and S. Ko, "Millimeter-wave 5g antennas for smartphones: Overview and experimental demonstration," IEEE Transactions on Antennas and Propagation, vol. 65, no. 12, pp. 6250-6261, 2017.

[3] W. Menzel and A. Moebius, "Antenna concepts for millimeter-wave automotive radar sensors," Proceedings of the IEEE, vol. 100, no. 7 , pp. 2372-2379, 2012.
[4] D. L. Donoho et al., "Compressed sensing," IEEE Transactions on Information Theory, vol. 52, no. 4, pp. 1289-1306, 2006.

[5] M. D. Migliore, "A compressed sensing approach for array diagnosis from a small set of near-field measurements," IEEE Transactions on Antennas and Propagation, vol. 59, no. 6, pp. 2127-2133, 2011.

[6] M. D. Migliore, "Array diagnosis from far-field data using the theory of random partial fourier matrices," IEEE Antennas and Wireless Propagation Letters, vol. 12, pp. 745-748, 2013.

[7] G. Oliveri, P. Rocca, and A. Massa, "Reliable diagnosis of large linear arrays-a bayesian compressive sensing approach," IEEE Transactions on Antennas and Propagation, vol. 60, no. 10, pp. 4627-4636, 2012.

[8] A. Morabito, R. Palmeri, and T. Isernia, "A compressive-sensing-inspired procedure for array antenna diagnostics by a small number of phaseless measurements," IEEE Transactions on Antennas and Propagation, vol. 64, no. 7, pp. 3260-3265, 2016.

[9] E. J. Candes, "The restricted isometry property and its implications for compressed sensing," Comptes rendus mathematique, vol. 346, no. 9-10, pp. 589-592, 2008.

[10] E. Candes and J. Romberg, "Sparsity and incoherence in compressive sampling," Inverse problems, vol. 23, no. 3, p. 969, 2007.

[11] R. Obermeier and J. A. Martinez-Lorenzo, "Sensing matrix design via mutual coherence minimization for electromagnetic compressive imaging applications," IEEE Transactions on Computational Imaging, vol. 3, no. 2, pp. 217-229, 2017.

[12] M. E. Eltayeb, T. Y. Al-Naffouri, and R. W. Heath, "Compressive sensing for millimeter wave antenna array diagnosis," IEEE Transactions on Communications, vol. 66, no. 6, pp. 2708-2721, 2018.

[13] C. Xiong, G. Xiao, Y. Hou, and M. Hameed, "A compressed sensingbased element failure diagnosis method for phased array antenna during beam steering," IEEE Antennas and Wireless Propagation Letters, vol. 18, no. 9, pp. 1756-1760, 2019.

[14] M. Elad, Sparse and Redundant Representations: From Theory to Applications in Signal and Image Processing. Springer Publishing Company, Incorporated, 1st ed., 2010.

[15] I. Dhillon, R. Heath Jr, T. Strohmer, and J. Tropp, "Designing structured tight frames via alternating projection," IEEE Trans. Inform. Th, vol. 51, no. 1, pp. 188-209, 2005.

[16] T. Hong, H. Bai, S. Li, and Z. Zhu, "An efficient algorithm for designing projection matrix in compressive sensing based on alternating optimization,” Signal Processing, vol. 125, pp. 9-20, 2016.

[17] C. Yann, R. Loison, R. Gillard, M. Labeyrie, and J. Martinaud, "A new approach combining surrounded-element and compression methods for analyzing reconfigurable reflectarray antennas," IEEE Transactions on Antennas and Propagation, vol. 60, no. 7, pp. 3215-3221, 2012.

[18] T. Ince and G. Ögücü, "Array failure diagnosis using nonconvex compressed sensing," IEEE Antennas and Wireless Propagation Letters, vol. 15, pp. 992-995, 2015.

[19] D. Needell and J. A. Tropp, "Cosamp: Iterative signal recovery from incomplete and inaccurate samples," Applied and computational harmonic analysis, vol. 26, no. 3, pp. 301-321, 2009.

[20] S. Foucart and M.-J. Lai, "Sparsest solutions of underdetermined linear systems via $\ell_{q}$-minimization for $0<q \leq 1$," Applied and Computational Harmonic Analysis, vol. 26, no. 3, pp. 395-407, 2009.

[21] S. Boyd, N. Parikh, E. Chu, B. Peleato, J. Eckstein, et al., "Distributed optimization and statistical learning via the alternating direction method

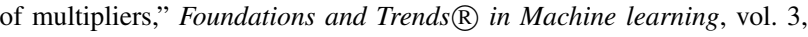
no. 1, pp. 1-122, 2011. 